\title{
Multilinguales
}

\section{L'hybridité de la langue française dans Un Sorcier blanc à Zangali de René Philombe}

The French language in Un Sorcier blanc à Zangali from predominance to hybridity

$$
\text { هجينية اللغة الفرنسية في ساحرأبيض في زانغالي لـ روني فيلومب }
$$

\section{Richard Tsogang Fossi}

\section{(2) OpenEdition}

\section{Journals}

\section{Édition électronique}

URL : http://journals.openedition.org/multilinguales/684

DOI : $10.4000 /$ multilinguales.684

ISSN : 2335-1853

\section{Éditeur}

Université Abderrahmane Mira - Bejaia

Référence électronique

Richard Tsogang Fossi, «L'hybridité de la langue française dans Un Sorcier blanc à Zangali de René Philombe », Multilinguales [En ligne], 7 | 2016, mis en ligne le 31 décembre 2016, consulté le 17 septembre 2019. URL : http://journals.openedition.org/multilinguales/684; DOI : 10.4000/ multilinguales.684

Ce document a été généré automatiquement le 17 septembre 2019.

\section{c) 89}

Multilinguales est mise à disposition selon les termes de la Licence Creative Commons Attribution Pas d'Utilisation Commerciale - Pas de Modification 4.0 International 


\section{L'hybridité de la langue française dans Un Sorcier blanc à Zangali de René Philombe}

The French language in Un Sorcier blanc à Zangali from predominance to hybridity

هجينية اللغة الفرنسية في ساحرأبيض في زانغالي لـ روني فيلومب

\section{Richard Tsogang Fossi}

1 "Il est utopique de s'attendre à ce que les Eurafricains que nous sommes parlent le français de France ", déclarait René Philombe à Efoua-Zengue (1989: 186 ), pour répondre à une question relative à la propension des mots en langue ewondo ${ }^{1}$ dans ses œuvres. Cette réaction d'un prix Mottart ${ }^{2}$ de l'Académie française à l'égard de la langue française, introduite au Cameroun dans un contexte colonial, mais qui est devenue l'une des langues officielles du pays, explique les différentes transformations qu'elle subit par rapport au français dit «central » ou standard, dans les œuvres de certains écrivains camerounais.

En effet, loin de rejeter cette langue, pourtant langue du colonisateur, ces écrivains vont l'enrichir de leurs langues et de leurs cultures pour finalement en faire leur patrimoine.

2 "Le Français me colonise, je le colonise à mon tour », déclarait l'écrivain congolais Tchicaya U Tam'si $(1992: 14)^{3}$. Cette prise de position rend compte de la volonté d'agir sur la langue d'importation encore perçue comme instrument de colonisation. Face à l'impossibilité de s'en défaire dans le contexte postcolonial, Tchicaya U Tam'si, à l'instar d'autres écrivains francophones, décide de se l'approprier.

3 Ainsi la langue française va se muer progressivement en un moyen d'exprimer l'africanité dans une littérature libérée des contraintes linguistiques du français central et éloignée des réalités de la France, à travers l'expérimentation d'une nouvelle forme d'écriture: l'écriture hybride. Celle de Philombe est imprimée des marques de la culturelle locale. 
4 Notre contribution vise à explorer la manière dont cet écrivain s'accapare la langue française pour la faire «communiquer» avec une langue africaine. L'analyse portera sur le roman Un Sorcier blanc à Zangali (1969)4, qui illustre parfaitement, de notre point de vue, le processus d'hybridité de l'écriture tant au niveau linguistique que culturel, de la période postcoloniale.

5 Son roman est une sorte d'archéologie du fait colonial dont les couches se superposent comme un palimpseste. Les périodes convoquées sont celle de l'époque coloniale allemande (1884-1916), mais surtout la période charnière, entre 1914 et 1916, qui correspond à l'arrivée des Anglais et des Français au Cameroun lors de la Première Guerre mondiale.

6 Dans le roman USBZ, un missionnaire alsacien, le révérend père Marius, prend la lourde décision de se rendre, en $1915^{5}$, dans le très redouté village de Zangali où son prédécesseur aurait été mangé par la population. Il tient à évangéliser ce peuple, à le " civiliser », et à porter un coup sérieux au paganisme : "Ma décision intime était de ne pouvoir écrire qu'après avoir planté la croix du Christ à Zangali. (...) Je me trouve ici dans un pays totalement fermé aux bienfaits de la civilisation» (171). Après un parcours jonché $\mathrm{d}$ 'embûches, il parvient enfin à Zangali où il n'est pas le bienvenu.

7 L'intérêt de notre travail réside dans l'attention qui sera portée à deux éléments essentiels de l'écriture camerounaise hybride: son oralisation et le décentrement linguistique de la langue, ici la langue française.

\section{Oralisation de l'écriture littéraire}

8 L'oralisation de l'écriture consiste en l'insertion, dans l'écriture en langue française, d'éléments caractéristiques de la tradition orale africaine, dans des situations narratives similaires à celles qui sont les leurs dans la vie réelle. En d'autres termes, ces éléments sont narrativisés, intégrés dans la trame du roman sous forme de calques contextualisés dans la diégèse du roman. Emmanuel Kamgang note au sujet de la combinaison de l'oralité et de l'écriture qu' "il y a de nombreux collages d'énoncés traditionnels provenant des patrimoines oraux (proverbes, chants, contes et mythes) dont on rencontre des exemples dans tous les genres " $(2009: 187)$.

9 La restitution, à l'écrit, de ces "patrimoines oraux", à travers des techniques romanesques respectueuses de leur modalité orale, le recours à leurs passeurs culturels (griot, conteur, voyant/sorcier, initiateur,...) intégrés à la société de roman sont caractéristiques de ces œuvres.

\section{L'oralisation par les proverbes}

10 L'auteur de notre corpus intègre les proverbes dans le discours direct de certains personnages; d'où la première justification scripturaire de leur oralisation en texte.

11 L'intérêt accordé aux proverbes, notamment par Philombe, tient à leur fonction dans la société africaine puisqu'ils sont "un élément didactique important. Il[s] répond[ent] au besoin qu'éprouvent les hommes de communiquer leur expérience par la juxtaposition des images, des allégories» (Maxime Meto'o, 1989: 55). Cette fonction est d'autant plus justifiée que 
la somme des proverbes apparaît (...) comme une collection de sentences qui sert de référence aux membres d'un groupe d'hommes pour communiquer des préceptes essentiels à son organisation, ou rétablir la communication lorsque celle-ci est menacée d'interruption (idem).

12 Ainsi, les proverbes sont un des éléments fondamentaux des cultures orales africaines et leur insertion dans un texte écrit en français relève d'une volonté manifeste de les reconnaître et de les assumer comme tels, c'est-à-dire dans le sens décrit par Maxime Meto'o. En texte, en effet, le proverbe répond à de multiples motivations en situation de communication interactive : convaincre, expliciter, commenter, justifier, illustrer une réalité ou une situation, etc.

Dans USBZ, par exemple, le proverbe est convoqué comme argument d'autorité pour justifier, entre autre, une grande décision collective à prendre face à un dilemme dont l'issue menace la vie du clan : soit exécuter derechef le père Marius, ce qui reviendrait à se mettre à dos tous les ennemis de Zangali, soit le chasser simplement du village. Le représentant du chef Angula Beti be Nanga, qui semble voir dans la venue de ce "sorcier blanc " une conspiration de leurs ennemis, craint que le révérend Marius «ne soit qu'une simple clenchette qui, touchée par des mains imprudentes, laisserait ouvrir la porte sur une catastrophe "(116). Favorable plutôt à son expulsion pour l'éviter, il doit cependant présenter ses arguments, renforcés par des proverbes :

Dites-moi, a-t-on jamais vu un poisson fuir ses eaux natales pour aller vivre dans les arbres avec les singes? Jamais !... Et a-t-on jamais vu le palmier-raphia pousser au bord du fleuve Sanaga? Jamais !...Car, un proverbe dit fort bien: «Les buffles ensemble et les éléphants ensemble (116-117).

14 Ces propos sont "parlés », en ce sens qu'ils sont la parole proférée par quelqu'un en situation de communication. Mais ils sont également oralisés dans leur structure prosodique qui relève du style oral dont nous reconnaissons certains des procédés :

- le discours direct du locuteur avec interpellation explicite du destinataire en ouverture : « Dites-moi... » ;

- l'organisation parataxique des phrases qui préparent l'énoncé du proverbe et qui ne sont reliées que par une conjonction de coordination: "Dites-moi, a-t-on jamais vu un poisson fuir ses eaux natales pour aller vivre dans les arbres avec les singes? Jamais!»- « Et a-t-on jamais vu le palmier-raphia pousser au bord du fleuve Sanaga? Jamais!...»; la syntaxe parataxique est d'autant plus remarquable qu'elle peut décliner ses phrases à l'infini, mettant en valeur l'introduction du proverbe par une hypotaxe unique qui le présente comme la cause (unique) de l'ordre infini du monde : «Car, un proverbe dit fort bien : «Les buffles ensemble et les éléphants ensemble »;

- les reprises (répétitions) à des périodes régulières (en ouverture et en clausule) sur le modèle du proverbe lui-même : "Les buffles ensemble / et les éléphants ensemble » /" a-t-on jamais vu un poisson fuir ses eaux natales pour aller vivre dans les arbres avec les singes? Jamais !... » / «Et a-t-on jamais vu le palmier-raphia pousser au bord du fleuve Sanaga ? Jamais !... ».

Comme on peut le constater, ces reprises sont d'ordre à la fois syntaxique (reprise du même schéma phrastique), lexical (reprise des mêmes mots) et prosodique (reprise du même rythme).

L'oralisation provoque ainsi un effet de martellement qui participe de la force des arguments qui justifient le proverbe qui, à son tour, justifie la décision de chasser le père Marius de Zangali. De la façon dont celle-ci est présentée, elle ne semble dictée que par le proverbe qui règle l'ordre du monde - et non l'inverse - (« car un proverbe dit 
fort bien »), un ordre que l'arrivée de l'homme d'église, assimilé au colonisateur, bouleverse.

D'ailleurs un autre proverbe, sur le même schéma que celui-ci, décrit ce même bouleversement comme étant celui de la colonisation menée par «l'homme blanc»:

Transgressant cette loi divine, les hommes blancs ont abandonné leur terre à eux ! Ils ont traversé mille forêts, mille montagnes et mille nappes d'eau dans le seul but de venir nous chercher noise sur notre terre à nous ! (...) C'est la misère qui chasse

la martre de son terrier natal (117-118).

17 Nous retenons que, grâce à ces procédés d'oralisation, les proverbes, issus explicitement de la tradition orale, sont présents dans leur modalité orale d'origine. Peu importe leur authenticité avérée, il n'en demeure pas moins qu'ils se lisent comme des proverbes de la tradition orale.

\section{L'oralisation par les chants}

Les chants, à l'instar des proverbes, occupent une place importante dans la vie quotidienne des gens du village, dans le roman comme dans la réalité.

Dans notre corpus, il s'agit de chants de circonstance ou de chants sacrés. Les premiers sont de deux sortes: les chants collectifs adaptés à des situations collectives et les chantés-parlés individuels créés par certains personnages pour délivrer un message. Les seconds, à formes fixes, font partie de rituels sacrés.

Les chants collectifs de circonstance sont évoqués en général par le narrateur sous forme de thème. Ainsi, chargé de tonalités sombres, le chant rythme le travail forcé des gens du village pendant la colonisation :

Les populations, sommées d'abandonner la hutte familiale paisiblement cachée au cœur de la forêt, s'étaient installées au bord de cette route qui s'annonçait fort tumultueuse! (...). De tout cela, les villageois se souvenaient avec effroi et amertume. Et leur chant de travail montait en écho dans le ciel noir, troublant comme un de profundis jailli au fond d'un enfer. (49)

21 Le chant est identifié à travers la dénomination « chant de travail » au sein de laquelle «chant» reçoit un complément de nom qui spécifie son statut générique en tant qu'adapté à un type précis de circonstances.

Mais la tonalité du chant peut être sarcastique, quand il s'agit de recevoir le "Commandant Marius », comme à Pala, un village proche de Zangali :

Aussitôt tout Pala s'était mis à pied d'œuvre pour recevoir dignement le Commandant blanc. (...) On avait apprêté tam-tams et balafons, et tout le reste. Les chiens aboyaient. Les femmes poussaient des oyenga de gloire. Les instruments de musique lâchaient dans le ciel leurs meilleures vociférations. Le sol tremblait de rythmes dynamiques lorsque la voiture fit son entrée pour s'arrêter devant la « case de passage ». (72)

La rupture entre l'isotopie annoncée par « recevoir dignement » et son développement dans le sens opposé induit le sarcasme, lisible dans le parallélisme entre les phrases impliquant la symétrie et surtout l'équivalence entre :

- des sujets /+humain/, /+animal/, /+inanimé/: dans l'ordre, «les chiens... », «les femmes ... ", « les instruments ... ";

- des mots à valence explicitement opposée : aboy [ement] - oyenga de gloire - meilleures vociférations. 
La rupture isotopique, qui provoque la raillerie au niveau de la narration, marque un écart entre l'événement et ce qu'il est censé symboliser : la cérémonie de bienvenue pour accueillir le père Marius est un tohu-bohu.

Le chant de circonstance, traditionnel, collectif, les « oyenga de gloire » chantés par les femmes, est mêlé aux aboiements des chiens et aux «vociférations » des instruments, en signe d'opposition à la circonstance même.

5 Les chantés-parlés sont, au contraire, dictés par une situation particulière au niveau narratif. La gravité du chant entonné par Ombala Nga Ndugsa, le sage des sages de sa communauté, qui s'apprête à être fouetté sur la place publique, est préparé en amont :

Une tenue de palabre siège, présidée par le Mayor Dzomnigi ${ }^{6}$ en personne. Par suite d'une légère imprudence verbale, Ombala Nga Ndugsa est condamné à cette peine corporelle humainement inconcevable pour lui, par rapport à son âge et à sa distinction sociale. Devant ses enfants et les enfants de ses enfants en pleurs, le vieux Sage s'avance d'un pas grelottant d'effroi vers quatre mvulmetara ${ }^{7}$. (11)

Ombala Nga Ndugsa s'adresse en chantant au représentant militaire des autorités coloniales pour dénoncer non pas tant l'injustice que le manque de respect dont il est victime en tant qu'Ancien et Patriarche de sa communauté :

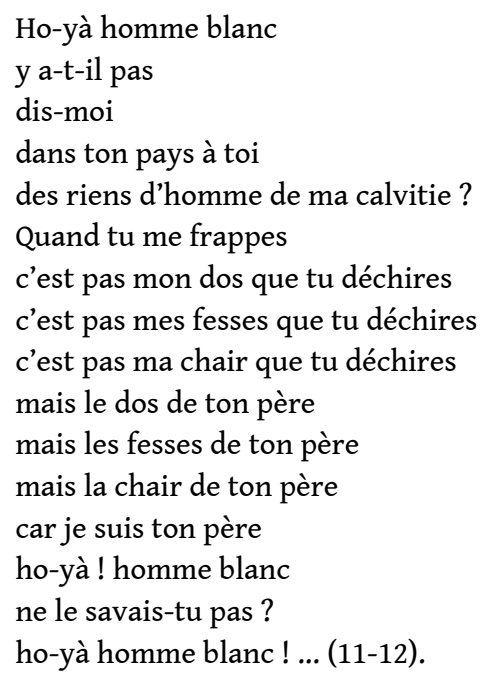

7 Le chant se présente comme la traduction mot à mot, en français, d'une langue locale désignée par l'interpellatif « ho-yà » dont les phonèmes sont translitérés.

De ce fait, le sens de certaines expressions telles que «des riens d'hommes de ma calvitie » est difficilement recouvrable. Mais elles sont tout à fait lisibles en contexte. Quelques lignes en amont, le narrateur délivre concomitamment la signification de la métonymie «calvitie » et le sens de cette scène de bastonnade : «ces scènes humiliantes, [de] ce manque total de respect envers les calvities les plus respectables du peuple! » (10).

Le sage interpelle l'officier allemand en l'identifiant par sa couleur, « ho-yà ! homme blanc », pour circonscrire le chant à l'ouverture et à la fermeture par une sorte de refrain.

Entre les deux refrains, la structure en chiasme croise « ma calvitie » et ses métonymies - mises en valeur par une construction clivée à la forme négative, « c'est pas... » - avec «ton père" (celui de "l'homme blanc») et les mêmes substituts métonymiques (« dos »- «fesses »- « chair »). 
31 Ce parallélisme convoque, par symétrie syntaxique, le «père » de l'« homme blanc » à la même place que l'homme noir en tant qu'implicitement entendu comme le vis-à-vis de l'« homme blanc». Cette superposition aboutit, à la fin du chant, à leur fusion dans une relation de cause à effet : « car je suis ton père ».

32 Ainsi le chant déplace la dénonciation de l'humiliation du terrain politique (politique coloniale) vers le terrain universel de l'humain et des relations intergénérationnelles dans un cadre familial ; un terrain chargé symboliquement dans la culture locale.

Fortement rythmé, par ses nombreuses répétitions strictement organisées au niveau rhétorique, le chant délivre une leçon de sagesse à même d'être proférée devant et pour les autorités coloniales.

La troisième catégorie est celle des chants sacrés. Les rituels s'accompagnent toujours de chants qui leur sont spécifiques et qui participent de ces rituels. C'est le cas également, dans le roman de Philombe, du rite tsogo, par exemple, que l'auteur reprend fidèlement de la tradition orale, comme nous avons eu à le constater à travers la lecture de certains documents authentiques ${ }^{8}$ :

Alors, le prêtre officiant se leva seul devant l'assistance et ouvrit le rite sans attendre. L'heure est propice. / Ô pères de nos pères! Ô pères des pères de nos pères! faites que nos tristes cris parviennent à vos oreilles! Les vieux arbres périssent, mais les jeunes pousses les remplacent, n'est-ce pas ?/ - Heueueu! gronda puissamment l'assistance./ - Descendent avec le courant du fleuve sortilèges et maladies des fantômes, n'est-ce-pas ?/- Heueueu ! Mort aux morts et vie aux vivants, n'est-ce-pas ? / - Heueueu! / (...) Son escarcelle claquant sur son dos, le prêtre marcha à pas lents sur le rivage, s'arrêtant là, et plantant à chaque arrêt, des oignons sauvages ou un gui afin d'éloigner les mauvais esprits. Après avoir craché neuf fois en direction du fleuve, il s'installa devant une énorme pierre. Aussitôt une odeur nauséeuse empoisonna littéralement l'air. Toutes les femmes poussèrent un vaste carillon de youyous; puis elles entonnèrent un chant qui mettait en garde quiconque sentait le besoin de se boucher le nez.(...) Ayant terminé cette opération (...), il s'accroupit et creusa un trou dans le sol. Il le remplit du mélange repoussant. (...) Le prêtre remua le tout avec sa main qu'il lécha délicieusement en grand spectacle, au milieu de nouveaux youyous des femmes. (...)L'on entendait l'un s'accuser publiquement de vol, un autre d'empoisonnement, un troisième d'inceste ou d'intentions criminelles, ou de maléfices, ou de songes préjudiciables à un parent. Bref, toutes les souillures du peuple s'étalaient aux oreilles de tout le monde, dans une incroyable atmosphère de quiétude pieuse, sous une tornade de huées réprobatrices. (...) / Heu-heu tsogo mana / Heu-heu tsogo mana /Nge ongawoe/ Okarege na ongadzib (...) / (oui, oui, fini le tsogo/ Oui, oui, fini le tsogo / Si tu as tué /Confesse que tu as tué...). (161-164)

Au regard des documents consultés, nous pouvons affirmer que le récit de ce rite de purification est retracé fidèlement, en ses différentes étapes. Il se déroule sous l'autorité du "prêtre » coutumier. Ses chants ont pour but de purifier la communauté des maux qui ont porté atteinte à son bien-être et à son harmonie. Au cours du rituel purificatoire rythmé par des chants, les membres de la communauté « presse[nt] [leur] ventre »/ au sens de [confessent leurs crimes].

Ce rite participe de l'oralisation de l'écriture grâce à ses deux moments les plus importants : son ouverture par les incantations du prêtre au style direct et sa fermeture par le refrain dans la langue d'origine (avec sa traduction en français). Nous remarquons que la narration, pourtant détaillée du rituel, ne s'attarde pas sur les confessions des pécheurs, qui varient d'un tsogo à un autre, mais sur ces éléments fixes, immuables. 
D'autres chants appartenant à d'autres rites sont aussi présents dans l'œuvre.

\section{Le style oralisé}

37 L'écriture subit l'influence de la tradition orale en s'inspirant aussi de son style, à travers la répétition d'un même mot, à intervalles réguliers, dont le parallélisme crée un effet rythmique de martellement et un effet de redondance au niveau du sens, comme dans l'exemple suivant: "On le voyait tout sourire quand il servait la messe, habillé comme un ange de vêtements sacrés, frappés de broderies sacrées, embaumés de senteurs sacrées » (66).

38 La répétition du même adjectif "sacré(e)s" à la même place, pour décrire le personnage d'Azombo, filleul et domestique du père Marius, pendant l'office, crée à la fois un effet rythmique de martellement et un effet de saturation de sens iconoclaste. Contrairement à sa fonction dans la tradition orale, sa visée est ici ironique, s'agissant de la ressemblance d'Azombo avec « un ange ».

Ce procédé est largement exploité dans le roman de René Philombe.

39 Le narrateur extradiégétique use également d'un style imagé en reprenant à son compte, dans un discours indirect libre, comme ici, les propos des gens du village de Béti-Belongo quant à l'accueil du père Marius :

Dès l'ouverture deux camps se trouvèrent en présence. L'un se réclamait de la modération, de la prudence. Par la bouche de Ntonga Mebenga, il soutenait que les arguments devaient être malaxés vingt fois et tamisés avant que ne soit tirée une conclusion définitive. Et même, il estimait qu'il était très tôt pour que l'on songeât à en tirer une et qu'un renvoi s'avérait indispensable. (115-116)

Prendre le temps de la réflexion se dit «Malaxe[r]» et «tamise[r] vingt fois » «les arguments » par emprunt métaphorique aux activités locales de subsistance.

Nous avons tenté jusqu'ici d'illustrer l'hybridité de l'écriture à travers son oralisation, c'est-à-dire la présence dans le roman de la tradition orale locale dont les exemples sont légion. Nous aimerions également le faire au niveau du décentrement proprement linguistique de la langue française.

\section{Le décentrement de la langue française}

41 Interrogeant la notion de "français standard", dans un article intitulé précisément «Le français standard et la norme: l'histoire d'un "nationalisme linguistique et littéraire" à la française ", Séverine Rebourcet (2008) note, qu'au niveau sociolinguistique,

Il faut, a fortiori, envisager ce concept par un raisonnement négatif, autrement dit, par élimination. Le français standard n'est pas le français régional mais n'est pas non plus le français oral, ni même le français populaire; ainsi, comme l'indique Françoise Gadet, "il prétend à la neutralité devant les genres discursifs" (Gadet 2003, p. 20). Si nous considérons, par exemple, la stylistique et les divers registres de langue, le français standard devient le français incarnant la limite entre ce qui est oral et informel, et ce qui est plus formel, plus soutenu et littéraire. (2008:109)

Une autre façon de s'attaquer, dans le roman, à cette "neutralité » du "français standard» ainsi défini, réside dans la traduction littérale en français d'expressions 
locales lexicalisées (ou données comme telles), la création lexicale sur un modèle préexistant et les interférences.

\section{La traduction littérale d'expressions lexicalisées}

43 Le roman fourmille d'expressions lexicalisées traduites mot à mot de la langue locale au français. Cette traduction littérale provoque une «localisation » du style et donc de la langue qui affichent les marques de la culture locale sans traduction. Le lecteur non initié doit se référer au seul contexte pour leur compréhension. En voici un exemple : Ebogo Nkodo, dont la maigreur le rapproche de la mort, est sommé de dénoncer le responsable de son état (les gens du village soupçonnent son frère cadet Elandi Nkodo Mevungu) :

Quand la terre était terre, hélas! Vit-on jamais ça? ... Et que les choses sont changées! [...] Et alors? Mais toi, le tout petit bambin de pas plus tard qu'hier, tu veux suspendre la cuillère, avec la tête couverte de cheveux et la bouche pleine de dents! Tu veux faire honte au peuple et souiller la mémoire de tes honorables morts! Ne crains-tu pas la colère des dieux? Non, cela ne sera pas! Presse ton ventre, le peuple rassemblé t'écoute ! (153)

Dans ce contexte, l'expression " presser son ventre » signifie « avouer » mais aussi et surtout «se confesser » parce que c'est ainsi que cela se traduirait en référence au rite tsogo auquel elle appartient. En effet, décrivant ce rite en langue française, Marie-Paule Bochet De Thé écrit $(1985: 246)$ :

Après un aparté avec les anciens, l'officiant, un vieillard au visage masqué, donne ordre à des enfants d'allumer des foyers aux quatre coins de l'endroit. Puis, au nom des dieux, il entre dans une grande colère et exhorte les participants à "presser le ventre" pour se purifier.

L'écriture du roman, qui décrit fidèlement le déroulement du rite tsogo (voir supra), lui emprunte ses expressions lexicalisées, comme elle le fait également à la langue locale pour bon nombre d'entre elles. Mais leur effet est l'inverse de celui des expressions lexicalisées en général : traduites mot à mot de la langue locale, elles rendent la langue française « étrange » en pointant leur origine.

\section{La création lexicale}

\section{Les noms « relocalisés » en ewendo}

Chez Philombe, certains noms chrétiens des personnages sont doublés d'un prénom local, comme celui de " Markus Abega » - [Markus - Marc ], moniteur évangéliste.

A cette composition qui signe la réinscription du nom dans la culture locale, s'ajoute en général son altération phonique par la langue: la consonne / $\mathrm{j} /$ réalisée en yod: "Yoanes Mbarga " ([Johann -Jean]), "Yacob Ayissi " ([Jacques]), "Yosef Essomba " ([Joseph]). On assiste ici à une interférence de trois langues à la fois, à savoir la reproduction des sonorités allemandes $(\mathrm{j}=\mathrm{yod})$, retranscrites dans le texte français mais prononcées en langue locale qu'est l'ewondo.

Cette altération est validée par le narrateur dans un discours indirect libre : « Le R. P. Marius parla d'une voix brisée par l'émotion. Il dit à son filleul de rester, d'aller chercher ses affaires le lendemain chez Yacob Ayissi, le cuisinier » (37). 
Cette altération locale des noms est d'ailleurs pointée comme telle par le père Marius en s'adressant aux gens du village :

Désormais, poursuivit le R. P. Marius, sachez que vous êtes devenus des enfants de Dieu. Demeurez-le jusqu'à la mort. Priez souvent vos saints patrons, Yosef et Maria ; ils ne vous abandonneront point. Priez-les souvent très souvent même! Et puis renoncez définitivement aux fétiches : ce sont de faux dieux! Servez le seul vrai Dieu tout puissant, et son fils unique Notre Seigneur Jésus-Christ. (34)

Quand il s'agit de renforcer leur foi chrétienne, il reprend les noms des saints dans leur prononciation locale ( vos saints patrons : Yosef et Maria »), mais quand il est impliqué ( «notre »), il prononce le nom sans altération de la consonne /j/ par exemple : « Notre Seigneur Jésus-Christ ».

Cette distinction signifie bien une relocalisation de la langue française par altération locale de son système phonique.

\section{Les mots (re)composés}

On trouve également dans le roman de René Philombe, des créations de mots calquées sur des mots français, mais recomposés pour signifier une réalité locale en langue ewondo. C'est le cas du mot " essuie-larmes » (38) qui signifie l'enfant unique, dont la venue au monde essuie figurativement/sèche les larmes des parents qui désespéraient d'avoir une progéniture. C'est le cas du couple Yosef Kuya et de Maria Tumbé, parents d'Etienne Azombo devenu filleul et domestique du père Marius.

50 Le mot recomposé garde dans sa mémoire sémantique le sens des expressions lexicalisées en français (" essuie-tout », " essuie-mains », " essuie-glace ») tout en se (re)chargeant au niveau sémantique grâce à sa reconfiguration dans son nouveau contexte.

51 Citons également la tournure interpellative locale " (Qu'importe), fils-de-mes-pères, (les représailles, même disproportionnées qui pourraient en résulter!) 》(118).

L'interpellation "fils-de-mes-pères" est destinée à gagner à sa cause les interlocuteurs dont le locuteur se rapproche en s'associant à eux dans une désignation collective par la lignée.

52 On rencontre également des surnoms flatteurs comme « Lance-d'Ivoire » (76), « Colèred'Orage » (idem), «Sang-de-Bataille » (idem), et bien d'autres encore, qui sont autant de traductions, dans la culture locale, d'éloges adressées à quelqu'un dans un but intéressé.

\section{Les interférences}

\section{Les interférences lexicales}

53 Selon le Dictionnaire de Linguistique (Larousse, 1973: 265), l'interférence se produit "quand un sujet bilingue utilise dans une langue-cible A ([ici le français]), un trait phonétique, morphologique, lexical ou syntaxique caractéristique de la langue B (langue locale de l'auteur])».

Le cas le plus courant est celui des interférences entre langue étrangère et langue maternelle, considérées par certains linguistes et didacticiens comme des « erreurs » et de ce fait, l'interférence est qualifiée de négative ${ }^{9}$. 
54 seulement intégrés au français, mais au système morphosyntaxique du français. En voici quelques-uns: "Il y avait des mintombà et des otombo-nnam qui auraient dû planer seuls; et il y avait des bitùga et des aloà qui auraient dû ramper seuls !... " "(8). / "Les femmes poussaient des oyenga de gloire » (72).

Nous constatons que l'intégration dans la langue française ne signifie pas leur récupération totale, puisque le substantif en langue locale, précédé du déterminant du français au pluriel («des»), ne porte pas la marque du pluriel en français, en l'occurrence le «s », par exemple. Quant à la marque du genre, elle est supportée par l'adjectif en français (« seuls »).

Par ce procédé, les auteurs transforment un phénomène d'interférence linguistique entre les langues, qualifié de fautif du point de vue de leur maitrise intrinsèque, en procédé d'écriture créative.

Les écrivains procèdent donc à un renversement de sa valeur : l'interférence lexicale, intégrée au français au niveau morphosyntaxique, par le biais de déterminants du français, est ainsi reconnue littérairement et donc valorisée, de même qu'est valorisée la langue locale ainsi convoquée dans le texte. Celle-ci l'est d'autant plus chez René Philombe, qu'elle s'impose souvent sans traduction et donc dans sa pleine étrangeté pour le lecteur non initié dont elle force l'attention.

\section{Les interférences phoniques}

Si les interférences lexicales imposent la présence de la langue locale dans le texte en français sans modalisation particulière, les interférences phoniques valorisent la langue locale par opposition aux langues européennes.

Le père Marius est ridicule quand il s'aventure malencontreusement à communiquer avec les gens du village dans « leur langue » dont il ignore les signes diacritiques :

Cela faisait toujours rire les indigènes quand ils entendaient le curé de Mvolyé parler leur langue. Dans sa bouche d'Européen, presque tous les mots devenaient une suite de contresens. Certes, il savait parfaitement que l'ewondo est une langue essentiellement à tons. Mais jamais, il ne parvenait à marquer l'accent tonique nécessaire qui indique la différence entre les homographes...Quand il croyait exprimer par exemple le goût (zam), c'est à la lèpre (zam) ou au raphia (zam) qu'il faisait penser! De même on pensait à la grande antilope (mvoe) quand il parlait d'amitié (mvoe) ou de paix (mvoe) ! (31)

Ces nombreuses ruptures d'isotopies sémantiques dues à la confusion phonique des « accents » sont d'autant plus amusantes pour les gens du village qu'elles se produisent à l'insu de l'énonciateur, provoquant des situations cocasses :

Alors, le R. P. Marius vérifie la liste des inscriptions des années précédentes. Il s'arrête devant un nom et, après avoir lu dans la colonne "observations", il s'adresse à la fille mère en grondant de sa voix nasillarde : Mengasili wo na olede ma ebonn dzoe! Ebonn dzoe! Ebonn dzoe ene ve? Ngaobele ebonn? La pauvre femme, toute éplorée et consumée par l'ardent désir de devenir chrétienne à n'importe quel prix n'hésite pas! Elle confie sans tarder son bébé à une voisine, puis elle se place devant le R. P. Marius les jambes largement écartées. Puis, joignant le geste à la parole, elle se découvre les fesses en braillant de façon théâtrale : « Ebonn dzam dzi, a Tara! (32)

L'accent qui différentie les deux « homographes » (ebonn: sexe / ebonn: amants) n'est pas perçu par le père Marius parce qu'il est spécifique à la langue locale. 
$\mathrm{Au}$ terme de cette brève analyse, nous retenons que l'écrivain de notre corpus, René Philombe, a trouvé le moyen d'exprimer son identité hybride. Pour lui, le choix d'une «écriture bi-culturelle » participe de sa réalité d'« écrivain eurafricain » dont l'univers est désormais fondamentalement hybride. Selon lui, il devient impossible de "vouloir exprimer les réalités négro-africaines avec les seuls mots de France» (Philombe, in EfouaZengue, 1989 : 186). Il en a fait l'illustration dans son roman en recourant à différents procédés d'intégration de sa culture orale d'origine dans la langue française.

\section{BIBLIOGRAPHIE}

BOCHET DE THÉ M.P., « Rites et associations traditionnelles chez les femmes bëti (Sud du Cameroun) », dans Jean-Claude Barbier (dir.), Femmes du Cameroun, mères pacifiques, femmes rebelles, Éditions Khartala-ORSTOM, 1985, pp. 245-279. Consulté sur le site : http:// horizon.documentation.ird.fr/exl-doc/pleins_textes/pleins_textes_7/b_fdi_03_05/23857.pdf Dictionnaire de Linguistique, Librairie Larousse, 1973.

EFOUA-ZENGUE R., « La langue française habillée d'obom. Entretien avec René Philombe », in Notre Librairie, n 99, Littérature camerounaise, oct-déc.1989, pp. 184-187.

« Entretien de Thierry Ngongang avec Alain Nganang », réalisé et diffusé par la chaîne de télévision STV 2, rediffusion du 29/12/2013.

KAMGANG E., « La littérature africaine face à la globalisation : une approche traductologique », in Kasereka Kavwahirehi (dir.), dans Imaginaire africain et mondialisation. Littérature et cinéma, L'Harmattan, Paris, 2009, pp. 183-209.

LABURTHE -TOLBA P., « Un tsógó chez les Eton », in Cahiers d'études africaines, vol. 15, n 59, Ed. EHESS, 1975. pp. 525-540. Consultable sur le site : http://www.persee.fr/docAsPDF/ cea_0008-0055_1975_num_15_59_2584.pdf

MAIGUENEAU D., Le contexte de l'œuvre littéraire : Énonciation, écrivain, société, Dunod, Paris, 1993.

METO’O M., « Proverbes Bulu », in Notre Librairie, n 99, 1989, pp. 54-58. Consulté sur le site : http:// journals.yu.edu.jo/jjmll/Issues/Vo2No2_2010PDF/4.pdf

ODEH A. et RABADI N., «L'analyse des erreurs en FLE chez des apprenants jordaniens et bahreïniens ", in Jordan Journal of Modern Languages and Literature - JJMLL - , vol. $2 \mathrm{~N}^{\circ} 2,2010$, pp. 163-177.

PHILOMBE R., Un Sorcier blanc à Zangali, Éditions Clé, Yaoundé, 1969.

REBOURCET S., « Le français standard et la norme : l'histoire d'un "nationalisme linguistique et littéraire" à la française ", in Communication, lettres et sciences du langage, vol. 2, No 1, Printemps 2008. Consultable sur le site : https://clsl.recherche.usherbrooke.ca/vol2no1/ rebourcet_vol2no1_2008.pdf 


\section{NOTES}

1. Langue du peuple Beti établi au Cameroun, plus particulièrement au centre et au sud du pays.

2. Prix annuel, créé en 1949 pour soutenir la création littéraire.

3. Cité par Jacques Chevrier, «Francophonie et littérature comparée, vers de nouvelles aventures ", in Bulletin de liaison et d'information de la société française de littérature générale et comparée, $\mathrm{N}^{\circ} 12$, printemps 1992, Numéro spécial : Littérature comparée et francophonie, pp. 9-22.

4. Noté USBZ dans la suite du texte.

5. Rappelons qu'en 1915, la Première Guerre mondiale entre puissances belligérantes faisait également rage au Cameroun.

6. Officier de l'armée allemande qui commande la population du village et dont « le nom, Dzomnigi, donnait des cauchemars » (10).

7. Milice sous les ordres de l'armée allemande.

8. Philippe Laburthe-Tolba, dans une étude intitulée « Un tsógó chez les Eton », se propose de « relater (...) le déroulement d'un des rares rituels qui aient survécu à l'action missionnaire chrétienne en pays Beti, le tsógó des Eton qui correspond au tsó des Ewondo. Avec la permission des personnes concernées je décrirai cette cérémonie telle qu'elle s'est passée en un lieu temps et milieu déterminés (...). Le chant est exécuté en solo par Ekodo; à la fin de chaque "verset ", l'assistance répond E-éé "d'accord ». En voici la traduction.

Chant $n^{\circ} 1$ : Qui a tué dit qu'il a tué - d'accord! / Qui a vu tuer dit qu'il a vu tuer - d'accord!/ Qui a volé même un chien même un chat - dira qu'il a volé - d'accord!/ Quand il aura tout dit il aura la bénédiction (mëmbà) - d'accord! Une sorte de refrain parlé énumère ensuite en une litanie les mérites du tsógó qui apporte bénédiction, richesse, enfants, etc. Chaque fois la foule approuve Êé, ce qui donne : Tsógó mëmbà (« bénédiction ») - Êé !/

Tsógó akùmà (« richesse ») - Êé ! T Tsógó bón (« enfant ») - Êé ! (1975 : 525-531).

Consulté également au sujet de ce rite, Bochet M.P., « Rites et associations traditionnelles chez les femmes bëti (Sud du Cameroun)", dans Jean-Claude Barbier (dir.), Femmes du Cameroun, mères pacifiques, femmes rebelles, Editions Khartala-ORSTOM, 1985, pp. 245-279. Disponible sur le site :

<http://horizon.documentation.ird.fr/exl-doc/pleins_textes/pleins_textes_7/

b_fdi_03_05/23857.pdf>

9. A titre d'exemple, dans un article intitulé «L'analyse des erreurs en FLE chez des apprenants jordaniens et bahreïniens », A. Odeh et N. Rabadi écrivent "L'interférence négative de l'arabe donne naissance à des erreurs comme le monsieur, la madame, la mademoiselle» (2010:168). Consulté sur le site :

<http://journals.yu.edu.jo/jjmll/Issues/Vo2No2_2010PDF/4.pdf>

10. L'auteur évoque ici l'ordre traditionnel que l'ère coloniale a bouleversé : « Otombo-nnam » et « mintombà » renvoient aux maîtres dans la société ewondo, alors que «bitùga » et "aloà » renvoient aux esclaves.

\section{RÉSUMÉS}

Cette contribution se propose d'examiner l'usage de la langue française chez certains écrivains camerounais de la période de la postindépendance. Introduite à l'époque coloniale et imposée dans une logique de monopole, cette langue dite "standard» ou «centrale » est réappropriée, entre autre, par l'écriture littéraire qui lui imprime des marques culturelles locales et qui 
subvertit intentionnellement certaines de ses règles caractéristiques. A travers l'étude d'Un Sorcier blanc à Zangali de René Philombe (1969), nous montrons que le roman détourne la langue française pour exprimer une identité hybride dans le contexte postcolonial, à travers notamment deux processus : l'oralisation de l'écriture et le décentrement linguistique de la langue française.

This paper investigates the use of the French language as a tool of literary creativity in the postindependence era in Cameroon. The so-called "standard" and "pure" language, introduced in the colonial period as a means of colonial supremacy, is re-appropriated and subverted in some of its main rules by some writers of the former colonies, according to their rejected culture, which they intend to revive. The analysis of Un Sorcier blanc à Zangali of René Philombe (1969) shows how the author subdues the French language to the expression of his hybrid identity in the postcolonial context, through the use of elements from oral culture on the one hand, and the very linguistic de-centration of the French language on the other hand.

\section{INDEX}

Mots-clés : Littérature camerounaise, langue française, hybridité, René Philombe, un sorcier blanc à Zangali, oralisation, interférence

$$
\text { في زالدب الكاميروني, اللغة الفرنسية, الهجينية, روني فيلومب, ساحرأبيضفهرس الكلمات المفتاحية: }
$$

Keywords : Cameroon literature, the french language, hybridity, René Philombe, un Sorcier blanc à Zangali, oralisation, interference

\section{AUTEUR \\ RICHARD TSOGANG FOSSI}

Université de Dschang Dschang - Cameroun 